\title{
False-Negative Sentinel Node Biopsy
}

\author{
Omgo E. Nieweg, MD, PhD \\ Department of Surgery, The Netherlands Cancer Institute/Antoni van Leeuwenhoek Hospital, \\ Amsterdam, the Netherlands
}

The concept of the orderly progression of lymph node metastases is exciting and appeals to the surgical frame of mind. The momentum of the practice of lymphatic mapping may cause people to get carried away by their enthusiasm. It is thus important to keep an open eye for developments that may not live up to the expectations, and therefore, the paper from the Italian Melanoma Intergroup in this issue deserves attention. An important purpose of the study of 1313 patients with melanoma was to determine the ability of sentinel node biopsy to identify involved lymph node basins. The false-negative rate was found to be $14.4 \%$; this high rate was obtained despite a meticulous technique and a comprehensive quality control program. ${ }^{1}$ High false-negative rates have been reported before but have rarely caused a stir.

For patients with melanoma, there has long been consensus on the principal aspects of the technique of lymphatic mapping. The combination of preoperative lymphoscintigraphy and intraoperative use of both blue dye and a gamma ray detection probe provides the best retrieval rate. The sentinel node is identified in close to $100 \%$ of patients. The morbidity of the procedure is limited. The tumor status of the sentinel node has proven to be the most important prognostic factor and has been incorporated in the staging system. ${ }^{2}$ There is initial evidence that melanoma patients with lymph node metastases have an improved chance of survival if regional node dissection is done early on the basis of sentinel node status. ${ }^{3}$

Morton and Cochran, who introduced sentinel node biopsy, initially performed the procedure with routine regional node dissection for confirmation and found an encouraging false-negative rate of $5 \%{ }^{4}$ Confirmatory

(C) Society of Surgical Oncology 2009

Published Online: 12 June 2009

O. E. Nieweg, MD, PhD

e-mail: o.nieweg@nki.nl lymph node dissection was quickly abolished, and the false-negative cases were subsequently identified through observation of recurrences in the lymph node basin after a tumor-free sentinel node had been removed. Initially, many investigators looked favorably on the reliability of lymphatic mapping to detect nodal metastases, but the followup duration was too short for all recurrences to manifest. Also, many investigators calculated the rate of false-negative procedures over the entire group of patients or over the group of sentinel node-negative patients. ${ }^{5}$ This is not correct because one cannot miss an involved node in a patient who does not have involved nodes. Because only approximately $20 \%$ of the patients have lymph node metastases, this questionable way of calculating decreases the false-negative rate by a factor of 5 . Testori et al. correctly point out that the false-negative rate should be defined as the fraction of the patients with involved nodes that is missed by the procedure and becomes evident later on when the nodes become clinically detectable (Table 1). The false-negative rate is the counterpart of the sensitivity, which is defined as the proportion of node-positive patients (sentinel node-positive patients and patients with recurring disease) that is identified by sentinel node biopsy. Early publications from reputable institutions and cooperative groups around the world showed false-negative rates ranging from $16 \%$ to $38 \%$ when recalculated to comply with the above recommended definition. ${ }^{5}$ Even in the interim analysis of the Multicenter Selective Lymphadenectomy Trial I, the false-negative rate is $17.6 \% .^{3}$

These high false-negative rates are reason for concern and reflection. What may be the cause? Testori et al. mention the nuclear medicine physician, the surgeon, and the pathologist as sources of failure, but there are more. It may be that the concept of sequential dissemination does not always apply. We are dealing with a biological system with associated variability. It is conceivable that tumor cells may pass through a sentinel node and lodge in the 
TABLE 1 Definition of terms

SLN sentinel lymph node false-negative rate $=$ \{patients with negative SLN with recurrent disease $\}$ /

\{patients with negative SLN with recurrent disease + patients with positive SLN sensitivity $=\{$ patients with positive SLN $\} /$

\{patients with negative SLN with recurrent disease + patients with positive SLN next lymph node. Another cause may be that the lymphoscintigraphy is not $100 \%$ accurate in pointing out the sentinel node. The new and sensitive single-photon emission computed tomography/computed tomography method has demonstrated that there are more sentinel nodes than can be found via conventional lymphoscintigraphy. ${ }^{6}$ Lymph flow is known to be variable. It is possible that the sentinel node contains tumor that blocks the lymphatic channel. ${ }^{7}$ Scintigraphy and blue dye will then be diverted to a "neo" sentinel node that may not yet be involved. We know how fast lymph fluid travels through a lymph vessel, but little is know about the kinetics of melanoma cells in lymphatics. Thus, another reason for a false-negative result may be that tumor cells are still in transit at the time of the sentinel node biopsy.

A meta-analysis of published results of lymphatic mapping in breast cancer patients revealed that the falsenegative rate ranges between $0 \%$ and $3 \%$ in that disease, with a weighed combined sensitivity of $100 \% .^{8}$ An interesting question is why the sensitivity is so much better in breast cancer. Lymphatic mapping started later in breast cancer, and completion node dissection was performed for a longer period of time than in melanoma. As a result, there are relatively few breast cancer studies with a long duration of follow-up. The median follow-up in the above-mentioned meta-analysis was 34 months. ${ }^{8}$ Differences in the physiology of lymph flow may also explain part of the disparity in the false-negative rate. Lymph flow from the skin is more variable compared with drainage from the breast parenchyma. We know this from studies where lymphoscintigraphy was performed twice in the same patient and a different sentinel node was visualized in $10 \%$ to $15 \%$ of the melanoma patients, but in none of the breast cancer patients. ${ }^{9,10}$ This suggests that in a fair number of melanoma patients, not all sentinel nodes are collected. Preoperative ultrasonography was introduced to identify metastases that may not be palpable but are large enough to block the inflow of lymph fluid. ${ }^{7,11}$ Ultrasound may thus identify the very nodes that will fail to pick up the tracers and may pass unnoticed during lymphatic mapping, causing the false-negative results later on. Ultrasound proved to be a valuable to detect nonpalpable metastases in patients with breast cancer. Radiologists note that ultrasound is more difficult in melanoma-and indeed, the radiologists at my institution, who are so apt at finding lymph node metastases in breast cancer patients (sensitivity $21 \%$ ), fail dramatically when the disease is melanoma (sensitivity $4.7 \%$ ).

Differences in the biology of the two diseases may play a role as well. Breast cancer is often a more slowly growing disease, which means that it will take more time for recurrence to manifest. The median time to recurrence in the axilla has been reported to be more than 6 years. ${ }^{12}$ In melanoma, $80 \%$ of the recurrences have established themselves within 3 years. ${ }^{13}$ Breast cancer cells in lymph nodes more often cluster together, which makes them easier to spot by the pathologist. The smaller melanoma tumor clumps may also metastasize via lymphatic channels with a smaller diameter. Therefore, one may speculate that they can spread to additional lymph nodes via narrow collateral lymphatic vessels that may not be open all the time and may not be depicted on the lymphoscintigram. In breast cancer, the procedure is more forgiving for a number of reasons. Several retrospective and prospective studies suggest that only about a third of the breast cancer metastases in the axilla develop into clinically important disease. Data from the Multicenter Selective Lymphadenectomy Trial suggest that this percentage appears to be considerably higher in melanoma. ${ }^{3}$ The radiotherapy to the breast that is part of breast-conserving treatment is likely to clean up some of the involved nodes that surgeons overlook and leave behind in the adjacent axilla. The same can be said for the adjuvant systemic treatment that many of these patients receive.

A few changes have been made in the technique since its introduction. For instance, intraoperative palpation of the lymph node field was introduced to identify unstained, nonradioactive nodes that are suspicious because of their consistency or size.

Some recent studies with a follow-up duration exceeding 3 years show improved false-negative rates of approximately $10 \% .^{14-19}$

How many recurrences are acceptable? In melanoma, this question is more pressing than in breast cancer because the sentinel node procedure is an additional operation, compared with the preexisting situation in which clinically normal lymph node fields were generally observed. Most melanomologists think that the extra prognostic information and the recently established survival benefit in node-positive patients outweigh the limited morbidity and the substantial false-negative rate. Single-photon emission computed tomography/computed tomography and ultrasound may 
lower the false-negative rate in the future, but the variability of lymph drainage from the skin and the pathophysiology of the disease are factors that will hamper further reduction.

\section{REFERENCES}

1. Testori A, De Salvo GL, Montesco MC, et al. Clinical considerations on sentinel node biopsy in melanoma from an Italian multicentric study on 1313 patients (SOLISM-IMI). Ann Surg Oncol. (in press) DOI:10.1245/s10434-008-0273-8.

2. Gershenwald JE, Thompson W, Mansfield PF, et al. Multi-institutional melanoma lymphatic mapping experience: the prognostic value of sentinel lymph node status in 612 stage I or II melanoma patients. J Clin Oncol. 1999;17:976-83.

3. Morton DL, Thompson JF, Essner R, et al. Immediate versus delayed lymphadectomy in the management of primary melanoma. N Engl J Med. 2006;355:1307-17.

4. Morton DL, Wen D-R, Wong JH, et al. Technical details of intraoperative lymphatic mapping for early stage melanoma. Arch Surg. 1992;127:392-9.

5. Nieweg OE, Tanis PJ, De Vries JDH, Kroon BBR. Sensitivity of sentinel node biopsy in melanoma. J Surg Oncol. 2001;78:223-4.

6. Van der Ploeg IMC, Valdés Olmos RA, Nieweg OE, et al. The additional value of SPECT/CT in lymphatic mapping in breast cancer and melanoma. J Nucl Med. 2007;48:1756-60.

7. Leijte JAP, Van der Ploeg IMC, Valdés Olmos RA, Nieweg OE, Horenblas S. Visualization of tumor-blockage and rerouting of lymphatic drainage in penile cancer patients using SPECT/CT. J Nucl Med. (in press).

8. Van der Ploeg IMC, Nieweg OE, Van Rijk MC, Valdés Olmos RA, Kroon BBR. Axillary recurrence after a tumour-negative sentinel node biopsy in breast cancer patients: a systematic review and meta-analysis of the literature. Eur J Surg Oncol. 2008;34:1277-84.
9. Kapteijn BAE, Nieweg OE, Valdés Olmos RA, et al. Reproducibility of lymphoscintigraphy for lymphatic mapping in patients with cutaneous melanoma. J Nucl Med. 1996;37:972-5.

10. Tanis PJ, Valdés Olmos RA, Muller SH, Nieweg OE. Lymphatic mapping in patients with breast carcinoma: reproducibility of lymphoscintigraphic results. Radiology. 2003;228:546-51.

11. De Kanter AY, Van Geel AN, Paul MA, et al. Controlled introduction of the sentinel node biopsy in breast cancer in a multicentre setting: the role of a coordinator for quality control. Eur J Surg Oncol. 2000;26:652-6.

12. Wright FC, Walker J, Law CH, McCready DR. Outcomes after localized axillary node recurrence in breast cancer. Ann Surg Oncol. 2003;10:1054-8.

13. Martini L, Brandani P, Chiarugi C, Reali UM. First recurrence analysis of 840 cutaneous melanomas: a proposal for a follow-up schedule. Tumori. 1994;80:188-97.

14. Doting MH, Hoekstra HJ, Plukker JT, et al. Is sentinel node biopsy beneficial in melanoma patients? A report on 200 patients with cutaneous melanoma. Eur J Surg Oncol. 2003;28:673-8.

15. Vuylsteke RJ, van Leeuwen PA, Muller MG, et al. Clinical outcome of stage I/II melanoma patients after selective sentinel lymph node dissection: long-term follow-up results. J Clin Oncol. 2003;21:1057-65.

16. Estourgie SH, Nieweg OE, Valdés Olmos RA, Hoefnagel CA, Kroon BBR. Review and evaluation of sentinel node procedures in 250 melanoma patients with a median follow-up of 6 years. Ann Surg Oncol. 2003;10:681-8.

17. Fincher TR, McCarty TM, Fisher TL, et al. Patterns of recurrence after sentinel lymph node biopsy for cutaneous melanoma. Am J Surg. 2003;186:675-81.

18. Yee VS, Thompson JF, McKinnon JG, et al. Outcome in 846 cutaneous melanoma patients from a single center after a negative sentinel node biopsy. Ann Surg Oncol. 2005;12:429-39.

19. Zogakis TG, Essner R, Wang HJ, et al. Melanoma recurrence patterns after negative sentinel lymphadenectomy. Arch Surg. 2005;140:865-71. 